\title{
Information-Theoretically Secure Oblivious Polynomial Evaluation in the Commodity-Based Model
}

\author{
Rafael Tonicelli ${ }^{1}$, Anderson C. A. Nascimento ${ }^{1}$, Rafael Dowsley ${ }^{2}$, \\ Jörn Müller-Quade ${ }^{3}$, Hideki Imai ${ }^{4}$, Goichiro Hanaoka ${ }^{4}$, Akira Otsuka ${ }^{4}$ \\ 1 Department of Electrical Engineering, University of Brasilia, \\ Campus Darcy Ribeiro, 70910-900, Brasilia, Brazil. \\ E-mail: tonicelli@redes.unb.br, andclay@ene.unb.br \\ 2 Department of Computer Science and Engineering, University of California San Diego, \\ 9500 Gilman Drive, La Jolla, California 92093, USA. \\ E-mail: rdowsley@cs.ucsd.edu \\ 3 Institute of Cryptography and Security, Karlsruhe Institute of Technology, \\ Am Fasanengarten 5, 76128 Karlsruhe, Germany. \\ E-mail: mueller-quade@kit.edu \\ 4 National Institute of Advanced Industrial Science and Technology (AIST), \\ 1-18-13, Sotokanda, Chyioda-ku, 101-0021, Tokyo, Japan. \\ E-mail:\{h-imai,hanaoka-goichiro,a-otsuka\}@aist.go.jp
}

\begin{abstract}
Oblivious polynomial evaluation (OPE) consists of a two-party protocol where a sender inputs a polynomial $p(x)$, and a receiver inputs a single value $x_{0}$. At the end of the protocol, the sender learns nothing and the receiver learns $p\left(x_{0}\right)$. This paper deals with the problem of oblivious polynomial evaluation under an information-theoretic perspective, which is based on recent definitions of Unconditional Security developed by Crépeau et al. [11]. In this paper, we propose an information-theoretic model for oblivious polynomial evaluation relying on pre-distributed data, and prove very general lower bounds on the size of the pre-distributed data, as well as the size of the communications in any protocol. It is demonstrated that these bounds are tight by obtaining a round-optimal OPE protocol, which meets the lower bounds simultaneously. We present a natural generalization to OPE called oblivious linear functional evaluation.
\end{abstract}

Keywords: Information-theoretic cryptography, cryptographic primitives, oblivious polynomial evaluation, commodity-based model.

\section{Introduction}

\subsection{Secure Function Evaluation}

Assume the existence of $n$ players, $1, \ldots, n$; each player $i$ has a private input $x_{i}$, which is known only to him/her. Their goal is to collaboratively compute $f\left(x_{1}, \ldots, x_{n}\right)$ in such a way that no player has to reveal unnecessary information about his/her input. A protocol allowing two or more parties to achieve this goal and satisfying both the correctness and the privacy constraints is called a secure function evaluation protocol. The correctness constraint implies that the values the protocol returns are correct, even if some party in the system fails (i.e., the faulty party can choose his/her input to the function, but cannot force the protocol to output a wrong value as the result of the function evaluation); and the privacy constraint implies that the joint computation of $f\left(x_{1}, \ldots, x_{n}\right)$ does not reveal to each participant $i$ more information than what can be deduced from $f\left(x_{1}, \ldots, x_{n}\right)$ and his/her own private input $x_{i}$.

There are two main ways of defining the security of a cryptographic system: InformationTheoretic Security (also called Unconditional Security) and Computational Security. For the former no assumption is made about the computational power of the adversary. For the later, 
the security is defined in terms of an adversary with limited computational resources. In order to prove that a system is computationally secure, it is necessary to invoke certain unproven intractability assumptions, e.g., the hardness of computing the discrete logarithm. In contrast, information-theoretically secure systems do not rely on any hypotheses about the complexity of certain problems, but rely on physical assumptions, e.g., the existence of noisy channels. In spite of being considered less practical, information-theoretic security is a permanent and stronger definition of security. This work focuses on the oblivious polynomial evaluation problem from an information-theoretic point of view, and is based on formal definitions of unconditionally secure evaluation schemes proposed by Crépeau at al. [11] which corrected many drawbacks present in several ad-hoc definitions of security proposed in the past.

\subsection{Oblivious Transfer}

Oblivious Transfer (OT), a cryptographic primitive introduced by Rabin [29], is of particular interest in secure multi-party computation. It has been proven that any function can be evaluated unconditionally securely if oblivious transfer is available [21]. This property is called completeness. A useful variant of oblivious transfer is one-out-of-n string OT, which is denoted by $\left(\begin{array}{l}n \\ 1\end{array}\right)-\mathrm{OT}^{k}$. It allows a sender to send $n$ strings $\left(x_{0}, \ldots, x_{n-1}\right)$ of length $k$ to a receiver, who is allowed to learn one of them according to his choice $c$. This process is illustrated in figure 1 .

An OT protocol is said to be correct, if for honest players, the receiver obtains the desired output $x_{c}$ and both players do not abort the protocol. It is said to be private if the sender learns no information on the receiver's choice $c$, while the receiver gets information concerning at most one of the sender's inputs.

It has been proven that unconditionally secure OT is impossible to achieve without further assumptions. Traditional assumptions used with this purpose include noisy channels and correlations, in which case both efficient protocols $[9,10,23]$ and the theoretical limits $[19,1,24,28]$ have been studied. ${ }^{5}$ In an ingenuous approach, Rivest [30] demonstrated that the use of a trusted third party, who pre-distributes data to the players, also allows one to design unconditionally secure OT protocols.

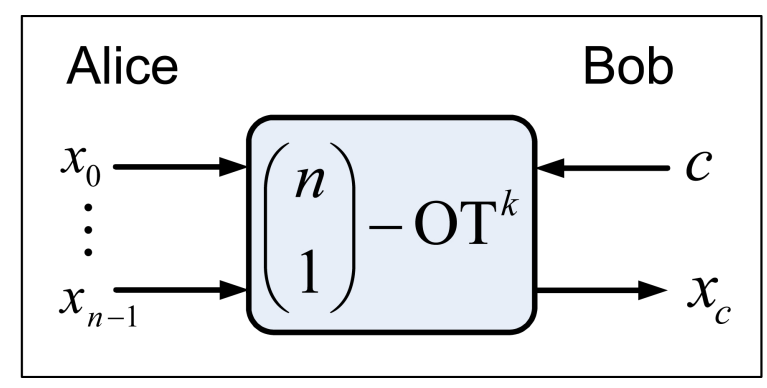

Fig. 1. One-out-of- $n$ string OT.

\subsection{Oblivious Polynomial Evaluation}

Oblivious Polynomial Evaluation (OPE) is a variant of Oblivious Function Evaluation and was introduced in [25]. Similarly to OT, OPE is a very useful tool for achieving secure distributed computations.

\footnotetext{
${ }^{5}$ In the computational security setting, OT can be based on various assumptions $[29,15,5,26,17,20,13,27$, $14]$.
} 
OPE is a two-party protocol where a sender (Alice) inputs a polynomial over a finite field and a receiver (Bob) inputs a single point of the same finite field. At the end of the protocol, Alice receives nothing and Bob should receive the polynomial input by Alice evaluated on the point chosen by him. This process is illustrated in figure 2. The protocol is secure if Bob evaluates the polynomial input by Alice on at most one point and Alice learns nothing on which point was chosen by Bob.

One can identify several applications where OPE is particularly valuable, such as protocols for private comparison of data and protocols for anonymity preservation. Some examples include: solutions for the list intersection problem, private metering, anonymous coupons, among others. Since its introduction in [25] OPE has been extensively studied. In [8] the problem of implementing OPE was efficiently reduced to that of achieving OT. Also, in [8] an information-theoretically secure protocol for implementing OPE was proposed. The security of that protocol was based on the trustiness of a third party which took an active role in the protocol execution.

In this paper, we analyze the problem of achieving unconditionally secure oblivious polynomial evaluation without using an active (on-line) trusted party.

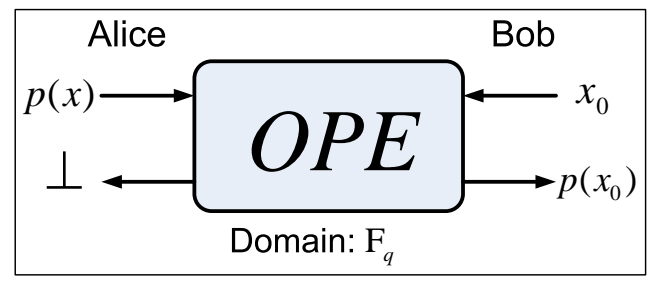

Fig. 2. Oblivious Polynomial Evaluation. Note that $\mathbb{F}_{q}$ denotes a finite field.

\subsection{Commodity-Based Cryptography}

Many security schemes demand an active server for intermediating the interactions among the participants of the protocol. Thus, the information exchanged among the participants will depend on the reliability and trustiness of the server during all the protocol execution. One alternative to this is the so-called Commodity-Based Cryptography, introduced by Beaver [2].

The protocols proposed in this paper rely on the commodity cryptographic model, where players buy cryptographic primitives from "off-line" servers, usually called trusted initializers (TIs). These primitives can be used later on to implement general cryptographic protocols. The commoditybased model was inspired on the Internet architecture, which is usually based on the "clientserver" paradigm. Once the primitives, or commodities as they are called by Beaver, are acquired, no further interactions between server and users are required (figure 3). Therefore, the servers need not to know the values which are computed by the players. Moreover, if several servers are available, they do not need to be completely trusted, that is, the system is secure even if some servers collude with the users of the commodities and/or among themselves. Another interesting feature of Beaver's model is that no interaction among the servers is required.

In this contribution, we show that the use of "off-line" servers provides very efficient and simple protocols for secure oblivious polynomial evaluation over a finite field.

Although this model was formalized just in [2], several independent works share the same flavor. We cite key-pre-distribution schemes [22], unconditionally secure bit commitments [30,7] and unconditionally secure digital signature schemes [18].

\subsection{Contributions and Related Works}

Seminal works on secure function evaluation and OPE were computationally secure. For instance, Naor and Pinkas proposed in [25] an OPE scheme which was based on the intractability assump- 


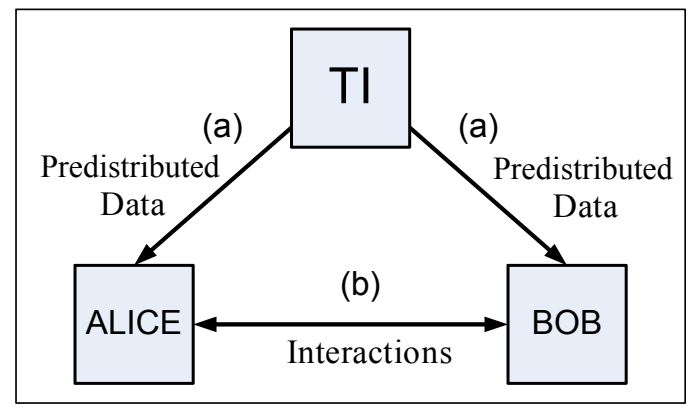

Fig. 3. Process (a) represents the setup phase and process (b) represents the interactions where no further intervention of the commodity server is needed.

tion of noisy polynomial interpolation. Later, Bleichenbacher and Nguyen demonstrated in [6] that this assumption could be less strong than expected and proposed a new intractability assumption based on the polynomial reconstruction problem. While the hardness of these problems remains an open question in the foundations of computer science, our OPE model is informationtheoretically secure, i.e., it is secure even against a computationally unbounded adversary and does not rely on unproven computational hypotheses.

Recently, Crépeau et al. [11] constructed a new formal definition of unconditional security, which is based on the ideal/real model paradigm, and established conditions for two-party secure function evaluation in a scenario where the players have infinite computational resources. By proving the security of our model, this work aims at revisiting the problem of oblivious polynomial evaluation from this new information-theoretic point of view.

We propose and solve the problem of implementing information-theoretically secure OPE in the commodity-based cryptography model [2]. Our solution is optimal in terms of communication complexity. We provide a model (section 2), bounds for the amount of memory which is required from players taking part in the protocol (section 3) and a construction which achieves these bounds, thus showing their tightness (section 4).

Finally, we propose a more general protocol called oblivious linear functional evaluation (OLFE) in section 5. In OLFE Alice inputs a linear functional while Bob evaluates this linear functional on a vector of his choice. As a side result of our bounds, we prove the optimality of oblivious transfer protocols proposed by Rivest [30] and Beaver [2].

\section{Definitions}

In this section, the general OPE model and important definitions used throughout the text are provided. These definitions include the security requirements for OPE realization and the scenarios in which our model is applicable.

\subsection{Notation}

In the following, we denote random variables by upper-case letters $(X)$ and their realizations by lower-case letters $(x)$. The set of values taken by a random variable is denoted by calligraphic letters $(\mathcal{X})$ and we use $|\cdot|$ to denote its corresponding cardinality $(|\mathcal{X}|)$. The Shannon entropy of a random variable $X$ is denoted by $H(X)$ and the mutual information of two random variables $X$ and $Y$ is denoted by $I(X ; Y)$. Similarly, $I(X ; Y \mid Z)$ and $H(X \mid Z)$ denote the conditional mutual information and the conditional entropy when conditioned on the random variable $Z$. 


\subsection{Security Definitions}

A two-party protocol consists of a program which describes a series of messages to be exchanged and local computations to be performed by the two parties. The protocol is said to halt if no more local computations or message exchanges are required. At the end of an execution of a protocol, each party emits an accept/reject message, depending on the messages he/she received and on the result of local computations.

Defining the security of a two-party protocol, where oblivious polynomial evaluation is an important special case, represents a challenging task. We consider scenarios where the parties are computationally unbounded and the existence of active and passive adversaries. An active (or malicious) adversary may change his/her behavior arbitrarily and cooperate in order to disrupt the correctness and privacy of the computation. On the other hand, a passive (or semi-honest) adversary is the one who follows the protocol, but may try to acquire more information than what he/she is allowed to know.

The definitions for information-theoretically secure two-party function evaluation used in this text are equivalent to the real/ideal model paradigm. In the ideal model, the parties are admitted to have access to a trusted third party, who would receive their private inputs, compute the outcome of the desired functionality $f$ and send to each party the corresponding output. In the real model, no trusted party for computing the functionality $f$ exists (possibly the parties have access to some functionality $g$ ), and the mutually distrustful parties should run some protocol to compute $f$. Intuitively speaking, if the real life protocol can emulate the ideal model, the protocol is said to be secure. In other words, a real life protocol is considered secure, if no adversary can cause more damage in a real execution than an ideal adversary can cause in an execution of the ideal protocol. Thus, if a protocol is secure according to this paradigm, an attack against the real life protocol has an effect similar to an attack against the ideal model, where the participants have only a black-box access to the desired functionality.

We shall now define when a protocol perfectly securely evaluates a function $f: \mathcal{X} \times \mathcal{Y} \rightarrow \mathcal{U} \times \mathcal{V}$. To accomplish this task, we will use the formalism and definitions of [11]. Let $x \in \mathcal{X}$ be the input of the first player and $y \in \mathcal{Y}$ the input of the second player. Consider also an additional auxiliary input $z \in\{0,1\}^{*}$ that can be potentially used by both players. For instance, this auxiliary input can be the data generated by previous protocol executions, or any other information that could give an illegal advantage to a dishonest party. Thus, an honest player will ignore this additional input. A $g$-hybrid protocol consists of a pair of algorithms $\Pi=\left(A_{1}, A_{2}\right)$ that can interact by means of two-way message exchange and have access to some functionality $g$. A pair of algorithms $\bar{A}=\left(\bar{A}_{1}, \bar{A}_{2}\right)$ is admissible for protocol $\Pi$ if at least one of the parties is honest, that is, if at least one of the equalities $\bar{A}_{1}=A_{1}$ and $\bar{A}_{2}=A_{2}$ is true. Note that no security is required when both parties are dishonest $\left(\left(\bar{A}_{1} \neq A_{1}\right) \wedge\left(\bar{A}_{2} \neq A_{2}\right)\right)$.

The Real Model. In the real model, the players have no access to a trusted intermediary and must compute the desired functionality by means of a $g$-hybrid protocol $\Pi=\left(A_{1}, A_{2}\right)$. Let $\bar{A}=\left(\bar{A}_{1}, \bar{A}_{2}\right)$ be an admissible pair of algorithms for the protocol $\Pi$. The joint execution of $\Pi$ under $\bar{A}$ in the real model,

$$
\operatorname{REAL}_{\Pi, \bar{A}(z)}^{g}(x, y)
$$

denotes the resulting output pair, given the input pair $(x, y) \in \mathcal{X} \times \mathcal{Y}$, the auxiliary input $z$ and the functionality $g$ used by the admissible pair $\bar{A}$.

The Ideal Model. In the ideal model, both players have access to a trusted third party to evaluate the functionality $f$. The trivial protocol $B=\left(B_{1}, B_{2}\right)$ is the protocol where both parties send their inputs to the functionality $f$ and output the values that the functionality $f$ outputs to them. The algorithms $\bar{B}_{1}$ and $\bar{B}_{2}$ of the protocol $\bar{B}=\left(\bar{B}_{1}, \bar{B}_{2}\right)$ receive the inputs $x$ and $y$, respectively, and the auxiliary input $z$. The algorithms send the values $x^{\prime}$ and $y^{\prime}$ to the trusted party, who returns the value $\left(u^{\prime}, v^{\prime}\right)=f\left(x^{\prime}, y^{\prime}\right)$. Finally, $\bar{B}_{1}$ and $\bar{B}_{2}$ output the values $u$ and $v$. Let $\bar{B}=\left(\bar{B}_{1}, \bar{B}_{2}\right)$ be an admissible pair of algorithms for $B$. The joint execution of $f$ 
under $\bar{B}$ in the ideal model on input pair $(x, y)$ and auxiliary input $z$, given by

$$
\operatorname{IDEAL}_{f, \bar{B}(z)}(x, y)
$$

represents the output pair that results from the interaction between $\bar{B}_{1}(x, z)$ and $\bar{B}_{2}(y, z)$ under the functionality $f$.

Figure 4 illustrates the abstraction of an admissible protocol $\bar{B}$ in the ideal model. At first, Alice receives input $(x, z)$ and Bob receives input $(y, z)$. Then, the parties produce the values $\overline{B_{1}^{I N}}(x, z)=\left(x^{\prime}, z_{1}\right)$ and $\overline{B_{2}^{I N}}(y, z)=\left(y^{\prime}, z_{2}\right)$. The parties send the inputs $x^{\prime}$ and $y^{\prime}$ to the trusted entity. The trusted entity performs the desired computation $f\left(x^{\prime}, y^{\prime}\right)=\left(u^{\prime}, v^{\prime}\right)$, and sends $u^{\prime}$ to Alice and $v^{\prime}$ to Bob. Upon having in their possession the outcomes $u^{\prime}$ and $v^{\prime}$ and the auxiliary inputs $z_{1}$ and $z_{2}$, Alice outputs $\overline{B_{1}^{O U T}}\left(u^{\prime}, z_{1}\right)=u$ and Bob outputs $\overline{B_{2}^{O U T}}\left(v^{\prime}, z_{2}\right)=v$. Note that when one of the parties is honest, he/she does not perform any modification on his/her inputs nor on his/her outputs. Additionally, auxiliary inputs are not used by honest participants.

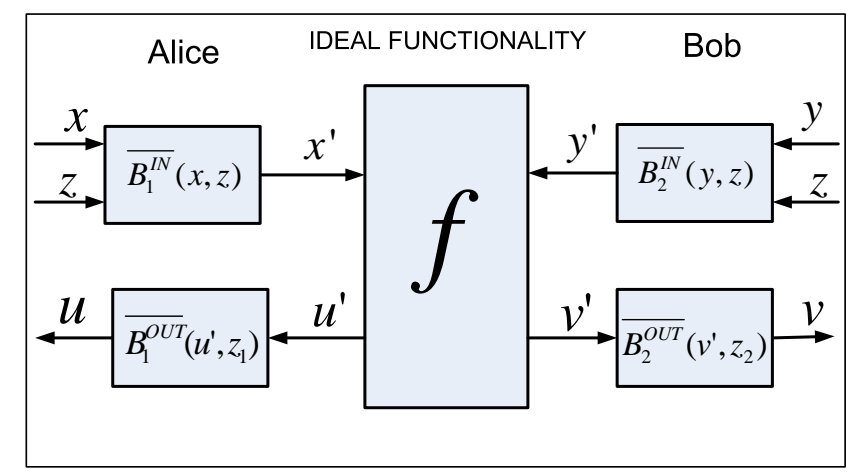

Fig. 4. Illustration of an admissible protocol $\bar{B}$ in the ideal model, where the parties have access to a trusted external entity.

Figure 5 exhibits the corresponding simplification of the model when one of the parties behaves honestly.
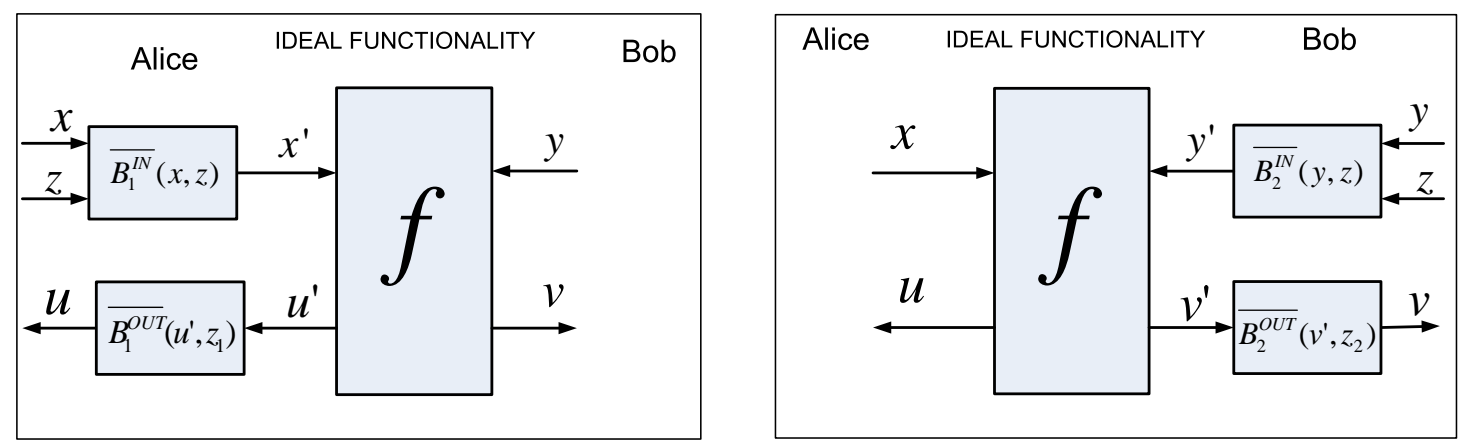

Fig. 5. Simplification of the ideal model for an honest Bob and an honest Alice, respectively. 
Definition 1 (Perfect Security). A g-hybrid protocol $\Pi$ evaluates a function $f$ perfectly securely if for every admissible pair of algorithms $\bar{A}=\left(\bar{A}_{1}, \bar{A}_{2}\right)$ in the real model for the protocol $\Pi$, there exists an admissible pair of algorithms $\bar{B}=\left(\bar{B}_{1}, \bar{B}_{2}\right)$ in the ideal model for the trivial protocol B, such that

$$
\operatorname{REAL}_{\Pi, \bar{A}(z)}^{g}(x, y) \equiv \operatorname{IDEAL}_{f, \bar{B}(z)}(x, y),
$$

for all input pair $(x, y) \in \mathcal{X} \times \mathcal{Y}$ and auxiliary input $z \in\{0,1\}^{*}$. Note that the symbol $\equiv$ denotes identical distributions.

Next we present a theorem from [11] which states the conditions for secure function evaluation.

Theorem 1. A protocol $\Pi$ is said to securely evaluate the deterministic functionality $f$ perfectly, if and only if for every pair of algorithms $\bar{A}=\left(\bar{A}_{1}, \bar{A}_{2}\right)$ that is admissible in the real model for the protocol $\Pi$ and for all inputs $(x, y) \in \mathcal{X} \times \mathcal{Y}$ and for all auxiliary input $z \in\{0,1\}^{*}, \bar{A}$ produces outputs $(U, V)$, such that the following conditions are satisfied:

- (Correctness) If both players are honest, we have

$$
(U, V)=f(X, Y)
$$

- (Security for Alice) If Alice is honest then there exist random variables $Y^{\prime}$ and $V^{\prime}$ such that $\left(U, V^{\prime}\right)=f\left(X, Y^{\prime}\right)$,

$$
I\left(X ; Y^{\prime} \mid Z Y\right)=0 \text {, and } I\left(U X ; V \mid Z Y Y^{\prime} V^{\prime}\right)=0 .
$$

- (Security for Bob) If Bob is honest then there exist random variables $X^{\prime}$ and $U^{\prime}$ such that $\left(U^{\prime}, V\right)=f\left(X^{\prime}, Y\right)$,

$$
I\left(Y ; X^{\prime} \mid Z X\right)=0, \text { and } I\left(V Y ; U \mid Z X X^{\prime} U^{\prime}\right)=0 .
$$

The security definitions are now applied to the oblivious polynomial evaluation problem. The ideal functionality $f_{\mathrm{OPE}}$ is denoted by

$$
f_{\mathrm{OPE}}\left(P, X_{0}\right):=\left(\perp, P\left(X_{0}\right)\right)
$$

such that $X_{0}, P\left(X_{0}\right) \in \mathbb{F}_{q}$, where $\mathbb{F}_{q}$ is a finite field, $P$ is a polynomial defined over $\mathbb{F}_{q}$ and $\perp$ denotes a constant random variable. The random variables $P$ and $X_{0}$ can have arbitrary probability distributions.

The application of our security definitions to the specific case of OPE yields to the following theorem.

Theorem 2. A protocol $\Pi$ realizes an OPE perfectly securely if and only if for every admissible pair of algorithms $\bar{A}=\left(\bar{A}_{1}, \bar{A}_{2}\right)$ for protocol $\Pi$ and for all inputs $\left(P, X_{0}\right)$ and auxiliary input $Z$, $\bar{A}$ produce outputs $(U, V)$ such that the following conditions are satisfied:

- (Correctness) If both players are honest, then

$$
(U, V)=\left(\perp, P\left(X_{0}\right)\right) .
$$

- (Security for Alice) If Alice is honest, then we have $U=\perp$ and there exists a random variable $X_{0}^{\prime}$, such that

$$
I\left(P ; X_{0}^{\prime} \mid Z X_{0}\right)=0 \text {, and } I\left(P ; V \mid Z X_{0} X_{0}^{\prime} P\left(X_{0}^{\prime}\right)\right)=0 .
$$

- (Security for Bob) If Bob is honest, then we have

$$
I\left(X_{0} ; U \mid Z P\right)=0 .
$$

Proof. We have to prove the equivalence between the privacy conditions for Bob in Theorems 1 and 2. This proof is analogous to the one presented in [11] for one-out-of- $n$ string OT.

According to Theorem 1 and using the notation introduced for OPE, we must have that

$$
I\left(X_{0} ; P^{\prime} \mid Z P\right)=0 \text {, and } I\left(P^{\prime}\left(X_{0}\right) X_{0} ; U \mid Z P P^{\prime} U^{\prime}\right)=0
$$


or equivalently,

$$
I\left(X_{0} ; P^{\prime} \mid Z P\right)+I\left(P^{\prime}\left(X_{0}\right) X_{0} ; U \mid Z P P^{\prime}\right)=0 .
$$

$P^{\prime}\left(X_{0}\right)$ is a function of $X_{0}$ and the polynomial $P^{\prime}$ so

$$
\begin{aligned}
I\left(P^{\prime}\left(X_{0}\right) X_{0} ; U \mid Z P P^{\prime}\right) & =I\left(X_{0} ; U \mid Z P P^{\prime}\right)+I\left(P^{\prime}\left(X_{0}\right) ; U \mid X_{0} Z P P^{\prime}\right) \\
& =I\left(X_{0} ; U \mid Z P P^{\prime}\right) .
\end{aligned}
$$

Then, the expression $I\left(P^{\prime}\left(X_{0}\right) X_{0} ; U \mid Z P P^{\prime}\right)=0$ is equivalent to $I\left(X_{0} ; U \mid Z P P^{\prime}\right)=0$.

Applying the chain rule for mutual information we obtain

$$
\begin{aligned}
I\left(X_{0} ; P^{\prime} \mid Z P\right)+I\left(X_{0} ; U \mid Z P P^{\prime}\right) & =I\left(X_{0} ; P^{\prime} U \mid Z P\right) \\
& =I\left(X_{0} ; U \mid Z P\right)+I\left(X_{0} ; P^{\prime} \mid Z P U\right) \\
& =I\left(X_{0} ; U \mid Z P\right) .
\end{aligned}
$$

The last equality follows from the fact that, in a secure OPE implementation, $P^{\prime}$ and $X_{0}$ have to be independent given $Z P U$. One can observe that, conditioned on $P$ and $Z$, Bob chooses his entry $X_{0}$ independently from $P^{\prime}$, the polynomial input actually supplied by Alice. Furthermore, we should require that the action of providing to the protocol a different input does not lead to any advantage to a malicious Alice. I.e., given that Alice knows $Z P U$, her knowledge on $P^{\prime}$ does not give her any additional information on Bob's input $X_{0}$. Mathematically, $X_{0}, Z P U$ and $P^{\prime}$ will form a Markov Chain:

$$
X_{0} \leftrightarrow Z P U \leftrightarrow P^{\prime} \Rightarrow I\left(X_{0} ; P^{\prime} \mid Z P U\right)=0 .
$$

Therefore, the security condition for a honest Bob is reduced to:

$$
I\left(X_{0} ; U \mid Z P\right)=0
$$

\subsection{Important Observation on the Auxiliary Inputs}

It is important to notice that the security conditions in theorems 1 and 2 must hold for all probability distributions of the inputs $(X, Y)$. More specifically, the security conditions have to be valid for any input distribution $P_{X Y \mid Z=z}$. Thus, all the underlying requirements are conditioned on the random variable $Z$. In order to prove the security of a protocol, one has to prove that the conditions are satisfied for all distributions $P_{X Y}$, omitting the random variable $Z$ in all the expressions.

Moreover, in [12], Crépeau and Wullschleger demonstrated that if a protocol is unconditionally secure against adversaries without auxiliary input, then it is also unconditionally secure against adversaries with auxiliary input.

As a consequence of this analysis, we will ignore the random variable $Z$ henceforth.

\subsection{Commodity-Based OPE}

In our model we have three players: Alice, Bob and Ted. We assume that the players are interconnected by private pairwise channels. The adversary is malicious and may deviate from the original protocol in an arbitrary way. Ted is a trusted center who pre-distributes some secret data to Alice and Bob during a setup phase, but does not take part in the protocol later on. The data received by Alice and Bob is denoted by the random variables $U_{a} \in \mathcal{U}_{a}$ and $U_{b} \in \mathcal{U}_{b}$, respectively. The pre-distributed data are chosen independently of the inputs.

In the computing phase, Alice and Bob interact in order to perform an oblivious polynomial evaluation. We assume that Alice and Bob are randomized players that are supplied by independent sources of randomness. By using this approach, we simplify notation. 
Note that in this way, all the messages generated by Alice and Bob are well-defined random variables, depending on the polynomial $P$ defined over $\mathbb{F}_{q}$ that Alice chose and on the evaluation point $X_{0} \in \mathbb{F}_{q}$ that Bob chose. The protocol can have many rounds of communication. Let the random variable $E$ denote all the messages sent by Bob and $R$ denote all the messages sent by Alice. As usual, we assume that the messages exchanged by the players are taken from $\{0,1\}^{*}$. So, assuming that both parties behave honestly, the views of Alice, VIEW $A$, and Bob, VIEW , after the protocol execution will be given by:

$$
\left\{\begin{array}{c}
\operatorname{ViEw}_{A}=\left\{U_{a}, P, E, R\right\} \\
\operatorname{ViEW}_{B}=\left\{U_{b}, X_{0}, E, R, P\left(X_{0}\right)\right\}
\end{array}\right.
$$

\section{Bounds}

\subsection{Remarks on the Adversarial Strategy}

More generally, the privacy conditions for an unconditionally secure two-party protocol can be translated into the following mathematical requirements:

$$
\left\{\begin{array}{l}
I\left(\mathrm{VIEw}_{A} ; V \mid \mathrm{VIEW}_{B}\right)=0, \text { thus } \operatorname{VIEw}_{A} \leftrightarrow \mathrm{VIEW}_{B} \leftrightarrow V . \\
I\left(\mathrm{VIEw}_{B} ; U \mid \mathrm{VIEW}_{A}\right)=0, \text { thus } \operatorname{VIEW}_{B} \leftrightarrow \mathrm{VIEW}_{A} \leftrightarrow U .
\end{array}\right.
$$

It means that, if a given protocol fulfills the preceding requirements, the optimal strategy for a corrupted player, who wishes to gain additional information on the other player's input, is to apply some arbitrary processing function (say $\varphi(\cdot)$ ) over his/her protocol view. So, we assume that the outputs, $U$ and $V$, of a corrupted Alice and a corrupted Bob are processed versions of their protocol views, $\mathrm{VIEW}_{A}$ and $\mathrm{VIEW}_{B}$, respectively:

$$
U=\varphi_{A}\left(\operatorname{VIEW}_{A}\right) \text { and } V=\varphi_{B}\left(\operatorname{VIEW}_{B}\right)
$$

\subsection{Computation of the Optimal Bounds}

In the sequel, we prove lower bounds on the memory and communication costs for oblivious polynomial evaluation in the commodity-based model. Since we are interested in OPE protocols that can be used with any input probability distribution, we assume that the input probability distribution has some properties $\left(P\right.$ and $X_{0}$ are independent and uniformly distributed random variables). To the specific task of calculating these lower bounds, we consider semi-honest adversaries. In section 4, we prove that these bounds are tight by presenting a protocol, secure against active cheating, that achieves all of them.

It is natural to think that, in our scenario, if Bob is given access to Alice's secret data $U_{a}$, he would be able to break the secrecy condition completely, that is he should be able to learn all the information about Alice's input $P$. We formally prove this fact in the next proposition.

Proposition 1. Bob learns all the information on $P$ if he is given access to Alice's pre-distributed data $U_{a}$ after completing a successful execution of oblivious polynomial evaluation. Mathematically, $H\left(P \mid E R U_{a} U_{b}\right)=0$.

Proof. Assume that a successful OPE execution, such $P=p(x)$ and $E R=e r$, has taken place. After obtaining Alice's pre-distributed data, Bob can try to compute $E R=e r$ for all the possible inputs. The correct input will produce a transcript equal to the one obtained during the protocol execution. Furthermore, the condition of security for Bob states that $I\left(X_{0} ; U \mid P\right)=0$. Since $E R U_{a}$ is part of Alice's view, $I\left(X_{0} ; E R U_{a} \mid P\right)=0$ and so

$$
H\left(X_{0} \mid E R U_{a} P\right)=H\left(X_{0} \mid P\right)=H\left(X_{0}\right),
$$


where the last equality follows from the fact that $P$ and $X_{0}$ are independent. It follows that for a given pre-distributed data, no two different polynomials should produce the same view, otherwise Alice would obtain knowledge on Bob's inputs (if two polynomials produce the same transcript, Bob's choice must be limited to the points where those polynomials coincide).

An equivalent result holds for Alice: if she is given access to the secret data that Bob received from Ted, she is able to completely break Bob's privacy condition, i.e., she learns $X_{0}$.

Proposition 2. Alice learns the point which was chosen by Bob if she is given access to Bob's pre-distributed data. Mathematically, $H\left(X_{0} \mid E R U_{a} U_{b}\right)=0$.

Proof. Assume a successful OPE execution, such that $P=p(x)$ and $E R=e r$. After this real execution is finished, we know from proposition 1 that $H\left(P \mid E R U_{a} U_{b}\right)=0$. Then, Alice can simulate Bob's inputs and determine those that are compatible with the transcript $E R=e r$. By the security condition for Alice, there cannot be two different values $\alpha_{1}$ and $\alpha_{2}$ compatible with the transcript, otherwise the correctness condition would allow Bob to discover $p\left(\alpha_{1}\right)$ and $p\left(\alpha_{2}\right)$ (violating Alice's security). So it follows that $H\left(X_{0} \mid E R U_{a} U_{b}\right)=0$.

We now prove another auxiliary result: namely, that the messages exchanged are independent of Alice's and Bob's inputs $P$ and $X_{0}$.

Proposition 3. In a secure commodity-based oblivious polynomial evaluation protocol, $I\left(P X_{0} ; E R\right)=$ 0. In particular, $H(P \mid E R)=H(P)$.

Proof. We start by rewriting the mutual information of interest:

$$
\begin{aligned}
I\left(P X_{0} ; E R\right) & =I\left(P X_{0} P\left(X_{0}\right) ; E R\right) \\
& =I\left(X_{0} P\left(X_{0}\right) ; E R\right)+I\left(P ; E R \mid X_{0} P\left(X_{0}\right)\right) \\
& =I\left(P\left(X_{0}\right) ; E R \mid X_{0}\right)+I\left(X_{0} ; E R\right)+I\left(P ; E R \mid X_{0} P\left(X_{0}\right)\right)
\end{aligned}
$$

Since the security for Bob states that $I\left(X_{0} ; U \mid P\right)=0$ and $E R$ is part of Alice's view, we have that $I\left(X_{0} ; E R \mid P\right)=0$ and so $I\left(X_{0} ; E R\right)=0$ because $P$ is independent of $X_{0}$. From the security condition for Alice it follows that $I\left(P ; V \mid X_{0} P\left(X_{0}\right)\right)=0$ and since $E R$ is part of Bob's view, we have that $I\left(P ; E R \mid X_{0} P\left(X_{0}\right)\right)=0$. Hence we get

$$
I\left(P X_{0} ; E R\right)=I\left(P\left(X_{0}\right) ; E R \mid X_{0}\right) .
$$

It remains to prove that the right hand side is 0 . Assume this were not the case.

Intuitively, we get a contradiction because $X_{0}$ is independent of $E R$, so Bob could go through the protocol and after receiving $R$ decide which value $P\left(X_{1}\right)$ he wants to obtain information about. Thus, he could not only learn his allotted $P\left(X_{0}\right)$ but also some more information, in violation of privacy for Alice.

The formal argument involves our technical condition on the distribution of $P$. Assume that $I\left(P\left(X_{0}\right) ; E R \mid X_{0}\right)>0$ for the purpose of getting a contradiction. Let $X_{1}=X_{0}+1$; in this way also $X_{1}$ takes on all values with positive probability, and the first part of our intuitive argument is valid: $I\left(P\left(X_{1}\right) ; E R \mid X_{1}\right)>0$, because $X_{1}$ can be generated by Bob independently of $E R$, just as $X_{0}$. Now we can estimate

$$
\begin{aligned}
I\left(P\left(X_{1}\right) ; X_{1}\right) & <I\left(P\left(X_{1}\right) ; X_{1}\right)+I\left(P\left(X_{1}\right) ; E R \mid X_{1}\right) \\
& =I\left(P\left(X_{1}\right) ; E R X_{1}\right) \\
& \leq I\left(P\left(X_{1}\right) ; E R X_{0} X_{1} P\left(X_{0}\right)\right) \\
& =I\left(P\left(X_{1}\right) ; X_{1} X_{0} P\left(X_{0}\right)\right)+I\left(P\left(X_{1}\right) ; E R \mid X_{0} X_{1} P\left(X_{0}\right)\right) \\
& =I\left(P\left(X_{1}\right) ; X_{1}\right)+0,
\end{aligned}
$$


a contradiction. We have only used standard identities and inequalities, except for the last line: there once more security for Alice was brought to bear, and the independence of $P\left(X_{1}\right)$ and $P\left(X_{0}\right)$ for $X_{0} \neq X_{1}$.

Hence our assumption was wrong, and the proposition is proved.

Now, we use the above propositions to prove a lower bound on the size of the data which is pre-distributed to Alice.

Theorem 3. In any commodity-based unconditionally secure oblivious polynomial evaluation protocol, the size of the data which is pre-distributed to Alice is as large as the size of the polynomial to be evaluated: $H\left(U_{a}\right) \geq H(P)$.

Proof. Consider $I\left(U_{a} ; P \mid E R U_{b}\right)$ : on the one hand we can rewrite it

$$
\begin{aligned}
I\left(U_{a} ; P \mid E R U_{b}\right) & =H\left(P \mid E R U_{b}\right)-H\left(P \mid E R U_{a} U_{b}\right) \\
& =H(P)-0,
\end{aligned}
$$

by propositions 1 and 3 and the fact that $P$ is independent of $U_{b}$. On the other hand,

$$
I\left(U_{a} ; P \mid E R U_{b}\right) \leq H\left(U_{a} \mid E R U_{b}\right) \leq H\left(U_{a}\right)
$$

which, put together with our previous identity, proves the theorem.

Another auxiliary result is actually just a corollary of proposition 3:

Proposition 4. In any commodity-based unconditionally secure oblivious polynomial evaluation protocol, $H\left(X_{0} P\left(X_{0}\right) \mid E R\right)=H\left(X_{0} P\left(X_{0}\right)\right)=H\left(X_{0}\right)+H\left(P\left(X_{0}\right) \mid X_{0}\right)$.

Proof. Proposition 3 states that $I\left(P X_{0} ; E R\right)=0$. By the data processing inequality, we thus have $I\left(X_{0} P\left(X_{0}\right) ; E R\right)=0$, which is just a reformulation of the claim.

Here, we show a bound on the size of the data pre-distributed to Bob.

Theorem 4. In any commodity-based unconditionally secure oblivious polynomial evaluation protocol, the size of the data which is pre-distributed to Bob is bounded by the following expression: for any $X_{0} \in \mathbb{F}_{q}, H\left(U_{b}\right) \geq H\left(X_{0}\right)+H\left(P\left(X_{0}\right) \mid X_{0}\right)$.

Proof. Consider the following expression:

$$
\begin{aligned}
I\left(U_{b} ; P\left(X_{0}\right) X_{0} \mid E R U_{a}\right) & =H\left(P\left(X_{0}\right) X_{0} \mid E R U_{a}\right)-H\left(P\left(X_{0}\right) X_{0} \mid E R U_{a} U_{b}\right) \\
& =H\left(X_{0}\right)+H\left(P\left(X_{0}\right) \mid X_{0}\right)-0
\end{aligned}
$$

using proposition 4 for the first entropy term, and proposition 2 (plus correctness of the protocol) for the second: $X_{0}$ is a function of $E, R, U_{a}$ and $U_{b}$, and all these data together determine the polynomial value $P\left(X_{0}\right)$ since $P$ is a function of these variables according to proposition 1 . On the other hand,

$$
I\left(U_{b} ; P\left(X_{0}\right) X_{0} \mid E R U_{a}\right) \leq H\left(U_{b} \mid E R U_{a}\right) \leq H\left(U_{b}\right),
$$

and with the previous identity the claim is proved.

We end this section with bounds on the size of the messages which have to be exchanged between Alice and Bob. 
Theorem 5. Let $R \in\{0,1\}^{*}$ and $E \in\{0,1\}^{*}$ be the random variables denoting, respectively, Alice's and Bob's communication during the computing phase. An unconditionally secure OPE protocol presents the following bounds:

$$
H(E) \geq H\left(X_{0}\right) \text { and } H(R) \geq H(P) .
$$

Proof. For the first bound, use proposition 2 for the first step in the following chain and then independence of $X_{0}$ and $R U_{a} U_{b}$ :

$$
\begin{aligned}
H\left(X_{0}\right) & =I\left(X_{0} ; E R U_{a} U_{b}\right) \\
& =I\left(X_{0} ; R U_{a} U_{b}\right)+I\left(X_{0} ; E \mid R U_{a} U_{b}\right) \\
& =I\left(X_{0} ; E \mid R U_{a} U_{b}\right) \leq H\left(E \mid R U_{a} U_{b}\right) \leq H(E)
\end{aligned}
$$

For the second one, use proposition 1 for the first step in the following chain and then independence of $P$ and $E U_{a} U_{b}$ :

$$
\begin{aligned}
H(P) & =I\left(P ; E R U_{a} U_{b}\right) \\
& =I\left(P ; E U_{a} U_{b}\right)+I\left(P ; R \mid E U_{a} U_{b}\right) \\
& =I\left(P ; R \mid E U_{a} U_{b}\right) \leq H\left(R \mid E U_{a} U_{b}\right) \leq H(R) .
\end{aligned}
$$

\section{An Optimal Construction}

In this section we present a construction based on polynomials over finite fields which matches the lower bounds we proved in the last section and is round optimal, thus proving their tightness. The intuition behind the protocol is that Ted distributes a random evaluation performed on a random polynomial to Alice and Bob during a setup phase. Later on, they will exchange messages to turn the random evaluation into the desired one. The protocol is described below.

\section{Protocol OPE}

\section{Setup Phase:}

- Ted selects uniformly at random a polynomial $s(x)$ of degree $n$ and a point $d \in \mathbb{F}_{q}$.

- Ted sends $s(x)$ to Alice and $\{d ; g=s(d)\}$ to Bob.

\section{Computing Phase:}

Alice's input: $p(x)$ of degree $n$.

Bob's input: $x_{0} \in \mathbb{F}_{q}$.

- Bob sends $t=x_{0}-d$ to Alice.

- Alice computes $f(x)=p(x+t)+s(x)$ and sends it to Bob.

- Bob computes $f(d)-g=p(d+t)+s(d)-s(d)=p\left(x_{0}\right)$, the desired output.

Theorem 6. The above stated protocol is a secure implementation of an oblivious polynomial evaluation protocol. Moreover, it is optimal regarding its space complexity. 
Proof.

(Correctness)

It is easily verifiable the correctness of the protocol. Considering both parties to be honest, we obtain

$$
f(d)-g=p(d+t)+s(d)-s(d)=p\left(x_{0}\right)
$$

which proves the correctness property.

(Security for Alice)

Let Alice be honest and $x_{0}^{\prime}=d+t$. Then,

$$
I\left(P ; X_{0}^{\prime} \mid X_{0}\right)=I\left(P ; D+T \mid X_{0}\right)=0
$$

since $D$ is independent of $P$.

Now we demonstrate that the second condition for Alice is satisfied. Let VIEW $B=\left\{D, G, T, F, X_{0}, X_{0}^{\prime}, P\left(X_{0}^{\prime}\right)\right\}$ be Bob's view of the protocol execution. Bob's output $V$ will be a processed version of VIEW $B$, consequently,

$$
\begin{aligned}
I\left(P ; V \mid X_{0} X_{0}^{\prime} P\left(X_{0}^{\prime}\right)\right) & \leq I\left(P ; \operatorname{VIEw}_{B} \mid X_{0} X_{0}^{\prime} P\left(X_{0}^{\prime}\right)\right) \\
& =I\left(P ; D G T F \mid X_{0} X_{0}^{\prime} P\left(X_{0}^{\prime}\right)\right) \\
& =I\left(P ; D G F \mid X_{0} X_{0}^{\prime} P\left(X_{0}^{\prime}\right)\right) \\
& =I\left(P ; D G \mid X_{0} X_{0}^{\prime} P\left(X_{0}^{\prime}\right)\right) \\
& =0 .
\end{aligned}
$$

Equation (1) follows from the fact that $t=x_{0}^{\prime}-d$ is a function of $d$ and $x_{0}^{\prime}$. Equation (2) follows from the fact that $f(x)=p(x+t)+s(x)$, where $S$ is uniformly random and independent of $P$. The last step results from the independence between the pre-distributed data $D, G$ and the polynomial $P$.

(Security for Bob)

Let Bob be honest and $f(x)=p^{\prime}(x+t)+s(x)$. Let $\operatorname{VIEw}_{A}=\left\{S, T, F, P, P^{\prime}\right\}$ be Alice's view of the protocol execution. Alice's output $U$ will be a processed version of $\operatorname{VIEW}_{A}$, consequently,

$$
\begin{aligned}
I\left(X_{0} ; U \mid P\right) & \leq I\left(X_{0} ; \operatorname{VIEW}_{A} \mid P\right) \\
& =I\left(X_{0} ; S T F P^{\prime} \mid P\right) \\
& =I\left(X_{0} ; S T F \mid P\right) \\
& =I\left(X_{0} ; S F \mid P\right) \\
& =0 .
\end{aligned}
$$

Equation (3) follows from the fact that $p^{\prime}(x)=f(x-t)-s(x-t)$, i.e., $P^{\prime}$ is fully determined by $S, T$ and $F$. Equation (4) follows from the fact that $t=x_{0}-d$, where $D$ is uniformly random and independent of $X_{0}$. The last step results from the fact that $f(x)=p^{\prime}(x+t)+s(x)$, where the data pre-distributed to Alice, $S$, is independent of Bob's input, $X_{0}$.

\section{(Optimality)}

Follows from the lower bounds obtained in theorems 3, 4 and 5 . 


\section{Oblivious Linear Functional Evaluation}

A linear functional $l$ on a vector space $\mathcal{W}$ is defined as a function $l: \mathcal{W} \rightarrow \mathbb{R}$, which satisfies the following properties:

$$
\left\{\begin{array}{c}
l(\mathbf{v}+\mathbf{w})=l(\mathbf{v})+l(\mathbf{w}) . \\
l(\alpha \mathbf{w})=\alpha l(\mathbf{w}) .
\end{array}\right.
$$

We generalize the previous OPE protocol to the case where Bob inputs $\mathbf{w} \in \mathcal{W}$ (vector space) and Alice inputs a linear functional $l \in \mathcal{W}^{*}$ (the dual vector space of linear functionals on $\mathcal{W}$ ). This task is called Oblivious Linear Functional Evaluation (OLFE). First, notice that evaluating a polynomial $p(x)=a_{0}+a_{1} x+a_{2} x^{2}+\ldots+a_{n} x^{n}$ on a point $x_{0}$ is the same as evaluating the linear functional $l=\left(a_{0}, a_{1}, \ldots, a_{n}\right)$ (as a row vector) on the (column) vector $\mathbf{w}=\left(1, x_{0}, x_{0}^{2}, \ldots, x_{0}^{n}\right)^{T}$. Thus OPE can be seen as a particular case of oblivious linear functional evaluation. This idea can be generalized to affine linear functionals, but we chose not to break the inherent beautiful symmetry via duality of the problem.

The choices of Alice and Bob are modeled by the random variables $L$ and $W$, which can have arbitrary probability distributions. The security conditions are analogous to the ones for OPE. The ideal functionality $f_{\mathrm{OLFE}}$ is denoted by

$$
f_{\mathrm{OLFE}}(L, W):=(\perp, L(W)) .
$$

Next theorem formalizes the conditions for a secure implementation of OLFE.

Theorem 7. A protocol $\Pi$ realizes an OLFE perfectly securely if and only if for every admissible pair of algorithms $\bar{A}=\left(\bar{A}_{1}, \bar{A}_{2}\right)$ for protocol $\Pi$ and for all inputs $(L, W)$ and auxiliary input $Z$, $\bar{A}$ produce outputs $(U, V)$ such that the following conditions are satisfied:

- (Correctness) If both players are honest, then

$$
(U, V)=(\perp, L(W)) .
$$

- (Security for Alice) If Alice is honest, then we have $U=\perp$ and there exists a random variable $W^{\prime}$, such that

$$
I\left(L ; W^{\prime} \mid Z W\right)=0 \text {, and } I\left(L ; V \mid Z W W^{\prime} L\left(W^{\prime}\right)\right)=0 .
$$

- (Security for Bob) If Bob is honest, then we have

$$
I(W ; U \mid Z L)=0 .
$$

Proof. We have to prove the equivalence between the privacy conditions for Bob in Theorems 1 and 7 . This proof is analogous to the previous one.

According to Theorem 1, we must have that

$$
I\left(W ; L^{\prime} \mid Z L\right)=0 \text { and } I\left(L(W) W ; U \mid Z L L^{\prime} U^{\prime}\right)=0 .
$$

or equivalently,

$$
I\left(W ; L^{\prime} \mid Z L\right)+I\left(L^{\prime}(W) W ; U \mid Z L L^{\prime}\right)=0 .
$$

$L^{\prime}(W)$ is a function of $W$ and the linear functional $L^{\prime}$ so

$$
\begin{aligned}
I\left(L^{\prime}(W) W ; U \mid Z L L^{\prime}\right) & =I\left(W ; U \mid Z L L^{\prime}\right)+I\left(L^{\prime}(W) ; U \mid W Z L L^{\prime}\right) \\
& =I\left(W ; U \mid Z L L^{\prime}\right) .
\end{aligned}
$$

Then, the expression $I\left(L^{\prime}(W) W ; U \mid Z L L^{\prime}\right)=0$ is equivalent to $I\left(W ; U \mid Z L L^{\prime}\right)=0$.

Applying the chain rule for mutual information we obtain

$$
\begin{aligned}
I\left(W ; L^{\prime} \mid Z L\right)+I\left(W ; U \mid Z L L^{\prime}\right) & =I\left(W ; L^{\prime} U \mid Z L\right) \\
& =I(W ; U \mid Z L)+I\left(W ; L^{\prime} \mid Z L U\right) \\
& =I(W ; U \mid Z L) .
\end{aligned}
$$


The last equality follows from the fact that, in a secure OLFE implementation, $L^{\prime}$ and $W$ have to be independent given $Z L U$. One can observe that, at the beginning of the protocol, Bob chooses his vector $W$ independently from $L^{\prime}$, the linear functional actually supplied by Alice. Furthermore, we should require that the action of providing to the protocol a different input does not lead to any advantage to a malicious Alice. I.e., given that Alice knows $Z L U$, her knowledge on $L^{\prime}$ does not give her any additional information on Bob's input $W$. Mathematically, $W, Z L U$ and $L^{\prime}$ will form a Markov Chain:

$$
W \leftrightarrow Z L U \leftrightarrow L^{\prime} \Rightarrow I\left(W ; L^{\prime} \mid Z L U\right)=0 .
$$

Therefore, the security condition for a honest Bob is reduced to:

$$
I(W ; U \mid Z L)=0
$$

Next, we present our construction of oblivious linear functional evaluation protocol. The intuition behind the protocol is similar to the one behind OPE. In the pre-distribution phase, Ted selects a random affine linear function and a random evaluation on the function and sends them to Alice and Bob, respectively. Subsequently, during the computing phase, Alice and Bob exchange information in order to obtain the desired result.

\section{Protocol OLFE}

\section{Setup Phase:}

- Ted selects with uniform randomness an affine linear function $m$ and a uniformly random $\mathbf{d} \in \mathcal{W}$.

- Ted transmits the function $m$ to Alice and the point $\{\mathbf{d} ; c=m(\mathbf{d})\}$ to Bob.

\section{Computing Phase:}

$$
\left\{\begin{array}{l}
\text { Alice's input: } l \in \mathcal{W}^{*} \\
\text { Bob's input: } \mathbf{w} \in \mathcal{W} .
\end{array}\right.
$$

- Bob sends $\mathbf{t}=\mathbf{w}-\mathbf{d}$ to Alice.

- Alice sends the function $n:=l+m+l(\mathbf{t})$ to Bob.

- Bob computes $n(\mathbf{d})-c=l(\mathbf{d})+m(\mathbf{d})+l(\mathbf{w}-\mathbf{d})-m(\mathbf{d})=l(\mathbf{w})$.

Theorem 8. The above stated protocol is a secure implementation of an oblivious linear functional evaluation protocol.

Proof.

(Correctness)

It is immediate to verify the correctness of the protocol. Considering both parties to be honest, we obtain

$$
n(\mathbf{d})-c=n(\mathbf{d})-m(\mathbf{d})=l(\mathbf{d})+l(\mathbf{w}-\mathbf{d})=l(\mathbf{w})
$$

(Security for Alice) 
Let Alice be honest and $\mathbf{w}^{\prime}=\mathbf{d}+\mathbf{t}$. As a consequence,

$$
I\left(L ; W^{\prime} \mid W\right)=I(L ; D+T \mid W)=0
$$

since $D$ is independent of $L$.

We shall now demonstrate that the second security condition for Alice also holds. Let $\mathrm{VIEW}_{B}=$ $\left\{D, C, T, N, W, W^{\prime}, L\left(W^{\prime}\right)\right\}$ be Bob's view of the protocol execution. Bob's output $V$ will be a processed version of $\mathrm{VIEW}_{B}$, consequently,

$$
\begin{aligned}
I\left(L ; V \mid W W^{\prime} L\left(W^{\prime}\right)\right) & \leq I\left(L ; \operatorname{VIEW}_{B} \mid W W^{\prime} L\left(W^{\prime}\right)\right) \\
& =I\left(L ; D C T N \mid W W^{\prime} L\left(W^{\prime}\right)\right) \\
& =I\left(L ; D C T \mid W W^{\prime} L\left(W^{\prime}\right)\right) \\
& =I\left(L ; D C \mid W W^{\prime} L\left(W^{\prime}\right)\right) \\
& =0 .
\end{aligned}
$$

Equation (5) follows from the fact that $n=l+m+l(\mathbf{t})$ is a function of $l, m$, and $\mathbf{t}$, such that $M$ is a random variable uniformly distributed and independent of $L$. Equation (6) follows from the fact that $\mathbf{t}=\mathbf{w}-\mathbf{d}$ is a function of $\mathbf{d}$ and $\mathbf{w}$. The last step is a consequence from the fact that the data pre-distributed to Bob, $D, C$ is independent of Alice's input $L$.

(Security for Bob)

Let Bob be honest. Let $\operatorname{VIEW}_{A}=\left\{M, N, T, L, L^{\prime}\right\}$. Alice's output $U$ will be a processed version of her view, $\operatorname{VIEW}_{A}$, consequently,

$$
\begin{aligned}
I(W ; U \mid L) & \leq I\left(W ; \operatorname{VIEW}_{A} \mid L\right) \\
& =I\left(W ; M N T L^{\prime} \mid L\right) \\
& =I(W ; M N T \mid L) \\
& =I(W ; M N \mid L) \\
& =0 .
\end{aligned}
$$

Equation (7) follows from the fact that $l^{\prime}+l^{\prime}(\mathbf{t})=m-n$, i.e., $L^{\prime}$ is fully determined by $M, N$, $L$ and $T$. Equation (8) follows from the fact that $\mathbf{t}=\mathbf{w}-\mathbf{d}$, where $D$ is uniformly random and independent of $W$. The last step results from the fact that $n=l^{\prime}+m+l^{\prime}(\mathbf{t})$, where the data pre-distributed to Alice, $M$, is independent of Bob's input, $W$.

\section{Conclusions}

In this paper we introduced and solved the problem of efficiently evaluating polynomials obliviously within the so-called commodity-based cryptography, as proposed by Beaver [2]. We proposed a model and then proved bounds on the amount of "commodities" which have to be pre-distributed by the trusted center, thus providing bounds for the amount of memory required by the players engaged in the protocol, as well as bounds on their communications. Then, we proved the tightness of our bounds by showing an explicit construction which meets them.

We also presented in this paper a definition of security for oblivious polynomial evaluation which is equivalent to the standard definition based on the real/ideal model paradigm. In the light of this new definition, we proved the unconditional security of our schemes. Finally, we proposed a generalization of oblivious polynomial evaluation: oblivious linear functional evaluation. 


\section{References}

1. R. Ahlswede, I. Csiszár. On Oblivious Transfer Capacity. ISIT 2007, pp. 2061-2064, 2007.

2. D. Beaver. Commodity-Based Cryptography (Extended Abstract). STOC 1997, pp. 446-455, 1997.

3. D. Beaver, S. Micali, and P. Rogaway. The Round Complexity of Secure Protocols. STOC 1990, pp. 503-513, 1990.

4. A. Beimel and T. Malkin. A Quantitative Approach to Reductions in Secure Computation. Theory of Cryptography Conference 2004, LNCS, vol. 2951, Springer-Verlag, pp. 238-257, 2004.

5. M. Bellare and S. Micali. Non-interactive oblivious transfer and spplications. CRYPTO89, pp. 547-557, 1990.

6. D. Bleichenbacher and P. Nguyen. Noisy Polynomial Interpolation and Noisy Chinese Remaindering. EUROCRYPT 2000, LNCS, vol. 1807, Springer-Verlag, pp. 53-69, 2000.

7. C. Blundo, B. Masucci, D.R. Stinson, and R. Wei. Constructions and Bounds for Unconditionally Secure Non-Interactive Commitment Schemes. Designs, Codes, and Cryptography, 26(1-3), pp. 97-110, 2002.

8. Yan-Cheng Chang, Chi-Jen Lu. Oblivious Polynomial Evaluation and Oblivious Neural Learning. ASIACRYPT 2001, LNCS, vol. 2248, Springer-Verlag, pp. 369-384, 2001.

9. C. Crépeau. Efficient Cryptographic Protocols Based on Noisy Channels. EUROCRYPT 1997, pp. 306-317, 1997.

10. C. Crépeau, K. Morozov, S. Wolf. Efficient Unconditional Oblivious Transfer from Almost Any Noisy Channel. SCN 2004, pp. 47-59, 2004.

11. C. Crépeau, G. Savvides, G. Schaffner, J. Wullschleger. Information-Theoretic Conditions for Two-Party Secure Function Evaluation. EUROCRYPT 2006, LNCS, 4004, Springer-Verlag, pp. 528-554, 2006.

12. C. Crépeau, J. Wullschleger. Statistical Security Conditions for Two-Party Secure Function Evaluation. ICITS 2008, LNCS, vol. 5155, Springer-Verlag, pp. 86-99, 2008.

13. R. Dowsley, J. van de Graaf, J. Müller-Quade, A. C. A. Nascimento. Oblivious Transfer Based on the McEliece Assumptions. ICITS 2008, pp. 107-117, 2008.

14. R. Dowsley, J. van de Graaf, J. Müller-Quade, A. C. A. Nascimento. Oblivious Transfer Based on the McEliece Assumptions. IEICE Transactions on Fundamentals of Electronics, Communications and Computer Sciences, E95-A (2), pp. 567-575, 2012.

15. S. Even, O, Goldreich, and A. Lempel. A randomized protocol for signing contracts. CRYPTO82, pp. 205-210, 1983.

16. O. Goldreich. Foundations of Cryptography, volume II: Basic Applications. Cambridge University Press, 2004.

17. I. Haitner. Implementing oblivious transfer using collection of dense trapdoor permutations. TCC 2004, pp. 394-409, 2004.

18. G. Hanaoka, J. Shikata, Y. Zheng, and H. Imai. Unconditionally Secure Digital Signature Schemes Admitting Transferability. ASIACRYPT 2000, LNCS, vol. 1976, Springer-Verlag, pp. 130-142, 2000.

19. H. Imai, K. Morozov, A. C. A. Nascimento. On the Oblivious Transfer Capacity of the Erasure Channel. ISIT 2006, pp. 1428-1431, 2006.

20. Y. T. Kalai. Smooth projective hashing and two-message oblivious transfer. EUROCRYPT 2005, pp. 78-95, 2005.

21. J. Kilian. Founding Cryptography on Oblivious Transfer. STOC 1988, pp. 20-31, 1988.

22. T. Matsumoto and H. Imai. On the Key Predistribution Systems. A Practical Solution to the Key Distribution Problem. CRYPTO 1987, LNCS, vol. 293, Springer-Verlag, pp. 185-193, 1988.

23. A. C. A. Nascimento, K. Morozov, H. Imai. Efficient Oblivious Transfer Protocols Achieving a Non-Zero Rate from Any Non-Trivial Noisy Correlation. ICITS 2007.

24. A. C. A. Nascimento, A. Winter. On the Oblivious-Transfer Capacity of Noisy Resources. IEEE Transactions on Information Theory 54(6), pp. 2572-2581, 2008. 
25. M. Naor and B. Pinkas. Oblivious Transfer and Polynomial Evaluation. STOC 1999, pp. 245-254, 1999.

26. M. Naor and B. Pinkas. Efficient oblivious transfer protocols. In 12th Annual ACM-SIAM Symposium on Discrete Algorithms, pp. 448-457, 2001.

27. C. Peikert, V.Vaikuntanathan, B. Waters. A framework for efficient and composable oblivious transfer. CRYPTO 2008, pp. 554-571, 2008.

28. A. C. B. Pinto, R. Dowsley, K. Morozov, A. C. A. Nascimento. Achieving Oblivious Transfer Capacity of Generalized Erasure Channels in the Malicious Model. IEEE Transactions on Information Theory 57(8), pp. 5566-5571, 2011.

29. M. O. Rabin. How to Exchange Secrets by Oblivious Transfer. Technical Report TR-81, Harvard, 1981.

30. R. Rivest. Unconditionally Secure Commitment and Oblivious Transfer Schemes Using Concealing Channels and a Trusted Initializer. Preprint available at http://people.csail.mit.edu/rivest/Rivest-commitment.pdf.

31. S. Wolf and J. Wullschleger. Oblivious Transfer is Symmetric. EUROCRYPT 2006, LNCS, vol. 4004, Springer-Verlag, 2006.

32. A.C. Yao. Protocols for Secure Computations. FOCS 1982, pp. 160-164, 1982. 